Pacific Journal of Mathematics

PRIME DIVISORS, ANALYTIC SPREAD AND FILTRATION 


\title{
PRIME DIVISORS, ANALYTIC SPREAD AND FILTRATIONS
}

\section{J. S. OKON}

\begin{abstract}
We show that a Noetherian ring $R$ is locally quasi-unmixed if and only if for every prime ideal $P \in \hat{A}^{*}(I), \mathrm{ht}(P)=l\left(I R_{P}\right)$. The analytic spread of an e.p.f., $l(f)$ is also defined and many of the known results for the integral closures of powers of an ideal are proven for the weak integral closures of the ideals in a strong e.p.f. Several characterizations are given of when a Noetherian ring $R$ is locally quasi-unmixed in terms of analytic spreads and integral closure of ideals. Several applications of these equivalences are given by showing when certain prime ideals are in $\hat{A}^{*}(f)$.
\end{abstract}

1. Introduction. Throughout this paper all rings will be assumed to be commutative with 1 .

We will show some relationships between the analytic spread of an ideal and its prime divisors. The influence of these relationships on the behavior of $\hat{A}^{*}(I)$ (see (2.2.3) for the definition) is also studied. In (2.6) it is shown that if $f=\left\{I_{n}\right\}$ is an essentially powers filtration (e.p.f., see (2.1.2)), in a Noetherian ring, then for a fixed $m^{\prime}, l\left(I_{m^{\prime}}\right)=l(f)$ (for all $n \geq 1$ ). (See (2.1.6) for the definition of $l$.) Our main Theorem (2.8), shows that a Noetherian ring $R$ is locally quasi-unmixed if and only if for each ideal $I$ in $R, P \in \hat{A}^{*}(I)$ implies $\mathrm{ht}(P)=l\left(I R_{P}\right)$. This is extended in (2.9) to e.p.f.'s. In (2.10)-(2.15), several equivalences are given to a Noetherian ring being locally quasi-unmixed; these are in terms of analytic spreads and integral closures of ideals. Several applications of these equivalences are given in (2.16)-(2.20) by showing certain prime ideals are in $\hat{A}^{*}(f)$.

2. Analytic spreads. We now give the definitions of the basic terms used in this paper.

(2.1) Definition. Let $R$ be a ring.

(2.1.1) A decreasing sequence of ideals $f=\left\{I_{n}\right\}_{n \geq 0}$ is said to be a filtration in case $I_{0}=R$ and for all $m$ and $n, I_{n} I_{m} \subseteq I_{n+m}$.

(2.1.2) (cf.) [1, Definition 2.14]. A filtration $f=\left\{I_{n}\right\}$ is said to be an essentially powers of an ideal filtration (e.p.f.) in case there exists $k>0$ such that $I_{n}=\sum_{1}^{k} I_{n-i} I_{i}$, for all $n \geq 1$, where $I_{i}=R$ if $i \leq 0$. 
(2.1.3) Let $I$ be an ideal in $R$. Then the Rees ring of $R$ with respect to $I, \mathcal{R}(R, I)$, is the ring $\Re(R, I)=R[u, t I]$ where $t$ is an indeterminant and $u=1 / t$. If $f=\left\{I_{n}\right\}$ is a filtration, then the generalized Rees ring of $R$ with respect to $f, R(R, f)$, is the ring $R(R, f)=R\left[u, t I_{1}, t^{2} I_{2}, \ldots\right](t, u$, as above).

(2.1.4) The integral closure of an ideal $I$ in $R$ is $\left\{x \in R \mid x^{n}+b_{1} x^{n-1}\right.$ $+\cdots+b_{i} x^{n-1}+\cdots+b_{n}=0$, where $\left.b_{i} \in I^{l}\right\}$. This set will be denoted by $I_{a}$.

(2.1.5) The weak integral closure of an ideal $I_{k}$ in a filtration $f=\left\{I_{n}\right\}$ is $\left\{x \in R \mid x^{n}+b_{1} x^{n-1}+\cdots+b_{i} x^{n-l}+\cdots+b_{n}=0\right.$, where $\left.b_{l} \in I_{k l}\right\}$. This set will be denoted by $\left(I_{k}\right)_{\alpha}$.

(2.1.6) Let $I$ be an ideal in $R$. Then the analytic spread of $I$, denoted $l(I)$, is defined as follows:

$$
l(I) \equiv \max \{\operatorname{alt}(\Re(R, I) /(u, M) \Re(R, I)) ;
$$

$M$ is a maximal ideal in $R$.

(2.1.7) Let $F=\left\{I_{n}\right\}$ be an e.p.f. in $R$. Then the analytic spread of $f$, denoted $l(f)$, is defined to be:

$$
l(f) \equiv \max \{\operatorname{alt}(\Re(R, f) /(u, M) \Re(R, f)) ;
$$

where $M$ is a maximal ideal in $R$.

(2.1.8) If $I$ is an ideal in $R$, then $V(I)$ denotes the smallest number of elements that generate $I$.

(2.1.9) A local ring $R$ is said to be quasi-unmixed in case, for every minimal prime ideal $z$ in the completion $R^{*}$ of $R, \operatorname{depth}(z)=\operatorname{alt}(R)$. A Notherian ring $R$ is said to be locally quasi-unmixed in case, for each prime ideal $P$ in $R, R_{P}$ is quasi-unmixed.

(2.1.10) An ideal $I$ in $R$ is said to be of the principal class in case $V(I)=\operatorname{ht}(I)$.

(2.1.11) An ideal $I$ is said to be integrally dependent on an ideal $B$ in case, $B \subseteq I \subseteq B_{a}$. An ideal $I$ is said to be weakly integrally dependent on an ideal $B_{n}$ in a filtration $f=\left\{B_{n}\right\}$ in case, $B_{n} \subseteq I \subseteq\left(B_{n}\right)_{\alpha}$

(2.2) REMARK. We will now state a few results on filtrations. Throughout, $R$ is a ring and $f=\left\{I_{n}\right\}$ is a filtration in $R$. The proofs are given in the cited references.

(2.2.1) [1, Proposition 2.18 and Theorem 2.20] and [10, (2.4.3)]. If $R$ is Noetherian, then $f$ is an e.p.f. if and only if there exist an integer $m>0$ such that for all $j \geq m, I_{m+j}=I_{m} I_{j}$. 
(2.2.2) [6, Corollary (2.8)]. Let $R$ be Noetherian. Fix $k>0$. If $f$ satisfies the condition:

(*) For all integers $m \geq m^{\prime}$ and $j>m^{\prime}, I_{m+j}=I_{m} I_{j}$, then the sets $B_{k}(n)=\operatorname{Ass}\left(I_{n} / I_{n+k}\right)$ are equal for all large $n$.

(2.2.3) [6, Corollary (2.8), Proposition (2.14) and Proposition (3.7)] and [2, Corollary 5]. If $f$ is a strong e.p.f. (in particular if $f$ is powers of an ideal) in a Noetherian ring $R$, then the sets $\hat{A}(n)=\left(\operatorname{Ass}\left(R / I_{n}\right)_{\alpha}\right)(=$ $\operatorname{Ass}\left(R /\left(I_{n}\right)_{\alpha}\right)$ for powers of an ideal) and $A(n)=\operatorname{Ass}\left(R / I_{n}\right)$ are constant for all large $n$. We denote the constant values by $\hat{A}^{*}(f)$ and $A^{*}(f)$ respectively. If $f=\left\{I^{n}\right\}$ then we will denote the sets by $\hat{A}^{*}(I)$ and $A^{*}(I)$ respectively.

(2.2.4) [5, Corollary (3.9)]. Let $R$ be a Noetherian ring and let $f=\left\{I_{n}\right\}$ be a strong e.p.f. Then for all $n \geq 1, \hat{A}^{*}\left(I_{n m^{\prime}}\right)=\hat{A}^{*}(f)$.

(2.3) Remark. Let $R$ be a Noetherian ring and let $f=\left\{I_{n}\right\}$ be an e.p.f. in $R$. The smallest integer $m$ satisfying $I_{m+j}=I_{m} I_{j}$ (see (2.2.1)) will be of particular importance throughout the paper. This integer will be denoted by $m^{\prime}$. A filtration satisfying condition (*) in (2.2.2) will be denoted as a strong e.p.f. The powers of an ideal $f=\left\{I^{n}\right\}$ is a strong e.p.f. with $m^{\prime}=1$.

Our first lemma proves a useful inequality relating the analytic spread of an ideal $I$ to the minimal number of generators of $I$. This inequality will be used throughout the chapter.

(2.4) LEMMA. Let $R$ be a Noetherian ring, let $I, P$ be ideals in $R$ such that $P$ is prime, and let $\Re=\Re\left(R_{P}, I R_{P}\right)$. Then, $l\left(I R_{P}\right) \leq V\left(I R_{P}\right) \leq V(I)$ (see (2.1.6) and (2.1.8)).

Proof. Since $R_{P}$ is local, $l\left(I R_{P}\right)=\operatorname{alt}\left(\Re /\left(u, P R_{P}\right) \Re\right)$. However, $\Re /\left(u, P R_{P}\right) \Re \cong\left(R_{P} / P R_{P}\right)\left[t\left(I R_{P}+\left(u, P R_{P}\right) /\left(u, P R_{P}\right)\right)\right]$, so

$$
\operatorname{alt}\left(\Re /\left(u, P R_{P}\right) \mathscr{R}\right) \leq V\left(I R_{P}\right) .
$$

Finally, it is clear that $V\left(I R_{P}\right) \leq V(I)$, so $l\left(I R_{P}\right) \leq V\left(I R_{P}\right) \leq V(I)$.

The next lemma shows that the Rees ring is integral over a certain subring. This will be used in the proof of (2.6).

(2.5) LEMMA. Let $R$ be a Noetherian ring and let $f=\left\{I_{n}\right\}$ be an e.p.f. in $R$. Let $m$ be large enough so that $R(R, f)=R\left[u, t I_{1}, \ldots, t^{m} I_{m}\right]$ and let $n$ be an arbitrary positive integer. Then $\Re(R, f)$ is integral over $R\left[u^{n m^{\prime}}, t^{n m^{\prime}} I_{n m^{\prime}}\right]$. 
Proof. It is sufficient to show that the elements of $t^{l} I_{l}$ are integral over $R\left[u^{n m^{\prime}}, t^{n m^{\prime}} I_{n m^{\prime}}\right]$, for $1 \leq i \leq m$. For this, let $a t^{i} \in t^{i} I_{l}$. Then $(a t)^{n m^{\prime}}=$ $a^{n m^{\prime}} t^{i n m^{\prime}}$ with $a^{n m^{\prime}} \in\left(I_{i}\right)^{n m^{\prime}} \subseteq I_{i n m^{\prime}}=\left(I_{n m^{\prime}}\right)^{l}$. Thus $\left(a t^{i}\right)^{n m^{\prime}} \in\left(t^{n m^{\prime}} I_{n m^{\prime}}\right)^{l}$. This shows that $a t^{i}$ is integral over $R\left[u^{n m^{\prime}}, t^{n m^{\prime}} I_{n m^{\prime}}\right]$. Therefore, $t^{l} I_{l}$ is integral over $R\left[u^{n m^{\prime}}, t^{n m^{\prime}} I_{n m^{\prime}}\right]$ for $1 \leq i \leq m$, and so $R(R, f)$ is integral over $R\left[u^{n m^{\prime}}, t^{n m^{\prime}} I_{n m^{\prime}}\right]$.

We now show that for a local ring, the analytic spread of an ideal of the form $I_{n m^{\prime}}$ in an e.p.f. $f=\left\{I_{n}\right\}$ is equal to the analytic spread of $f$.

(2.6) Lemma. Let $(R, M)$ be a local ring and let $f=\left\{I_{n}\right\}$ be an e.p.f. in $R$. Then for all positive integers $n, l\left(I_{n m^{\prime}}\right)=l(f)$.

Proof. Let $\Re=\Re(R, f)$ and let $\theta=\mathrm{R}\left[u^{n m^{\prime}}, t^{n m^{\prime}} I_{n m^{\prime}}\right]$, so $\Re$ is integral over $\theta$, by (2.5). By definition, $l(f)=\operatorname{depth}(M, u) \mathcal{R}$ and

$$
l\left(I_{n m^{\prime}}\right)=\operatorname{depth}(M, u) R\left[u, t I_{n m^{\prime}}\right]=\operatorname{depth}\left(M, u^{n m^{\prime}}\right) \mathcal{\vartheta} .
$$

Let $P$ be a minimum prime divisor of $(u, M) \Re$ such that $\operatorname{depth}(P)=l(f)$. Then $l(f)=\operatorname{alt}(\Re / P)=\operatorname{alt}(\theta /(P \cap \theta))$, since $\Re$ is integral over $\theta$. But $\left(M, u^{n m^{\prime}}\right) \Theta \subseteq P \cap \Theta$, so

$$
\operatorname{alt}(\theta /(P \cap \theta))=\operatorname{depth}(P \cap \theta) \leq \operatorname{depth}\left(M, u^{n m^{\prime}}\right) \theta^{\prime}=l\left(K_{n m^{\prime}}\right) .
$$

Thus $l(f) \leq l\left(I_{n m^{\prime}}\right)$. Now pick a minimal prime divisor $Q$ of $\left(M, u^{n m^{\prime}}\right) \theta$ such that $\operatorname{depth}(Q)=l\left(I_{n m^{\prime}}\right)$. Let $Q^{\prime}$ be a prime ideal in $R$ such that $Q^{\prime} \cap \vartheta=Q$. Then $Q^{\prime} \supseteq(M, u) \Re$, and so

$$
\begin{aligned}
l\left(I_{n m^{\prime}}\right) & =\operatorname{alt}(\Theta / Q)=\operatorname{alt}\left(\Re / Q^{\prime}\right)=\operatorname{depth}\left(Q^{\prime}\right) \\
& \leq \operatorname{depth}(M, u) \Re=l(f) .
\end{aligned}
$$

Thus $l\left(I_{n m^{\prime}}\right) \leq l(f)$, and so $l\left(I_{n m^{\prime}}\right)=l(f)$.

(2.7) REMARK. With the notation of (2.6), it would be interesting to know if $l\left(I_{n}\right)=l(f)$, for all $n \geq 1$.

The main theorem in this section shows that, for an ideal $I$ in a locally quasi-unmixed Noetherian ring $R$, if $P \in \hat{A}^{*}(I)$, then $\operatorname{ht}(P)=l\left(I R_{P}\right)$. This is a generalization of a result proved by Ratliff, for integral domains satisfying the altitude formula.

(2.8) THEOREM. (Cf. [12, Theorem 1].) Let $R$ be a Noetherian ring. $R$ is locally quasi-unmixed if and only if for every ideal $I$ in $R$ and for every prime ideal $P \in \hat{A}^{*}(I), \operatorname{ht}(P)=l\left(I R_{P}\right)$. 
Proof. Assume that for every ideal $I$ in $R$, if $P \in \hat{A}^{*}(I)$, then $\operatorname{ht}(P)=l\left(I R_{P}\right)$. Then to show that $R$ is locally quasi-unmixed it is sufficient to show that for each ideal $I$ of the principal class, $\left(I^{n}\right)_{a}$ is height unmixed for every integer $n \geq 1$, by [8, (2.29)]. For this, let $I$ be an ideal of the principal class and let $P$ be a prime divisor of $\left(I^{n}\right)_{a}$ for some $n \geq 1$. This implies $P \in \hat{A}^{*}(I)$, by $[6,(3.6 .1)]$ and $(2.2 .3)$, so by the hypothesis and (2.4),

$$
\operatorname{ht}(P)=l\left(I R_{P}\right) \leq V\left(I R_{P}\right) \leq V(I)=\mathrm{ht}(I) \leq \mathrm{ht}(P) .
$$

This proves the implication.

For the reverse implication, assume $R$ is locally quasi-unmixed. Let $I$ be an ideal in $R$ and assume $P \in \hat{A}^{*}(I)$. Then, with $\Re=\mathcal{R}(R, I)$, there exists a prime divisor $Q^{\prime}$ of $u \Re^{\prime}$ such that $Q^{\prime} \cap R=P$, by [11, Corollary 3]. Since $Q^{\prime}$ contains the regular element $u$, ht $\left(Q^{\prime}\right)=1$. Let $z^{\prime}$ be a minimal prime divisor in $\Re^{\prime}$ such that $z^{\prime} \subset Q^{\prime}$, let $Q=Q^{\prime} \cap \Re$, and let $z=z^{\prime} \cap \Re$. Also, let $z_{0}=z \cap R$. We now show that $\operatorname{ht}(Q / z)=1$.

For this, since $z=z_{0} R[t, u] \cap \Re$, we have

$$
\Re / z=\Re /\left(z_{0} R[t, u] \cap \Re\right) \cong \Re\left(R / z_{0},\left(I+z_{0}\right) / z_{0}\right),
$$

by [13, Lemma 1.1]. Therefore $R / z$ satisfies the altitude formula, since $R / z_{0}$ does (since $R$ is locally quasi-unmixed). Thus, since $\left(Q^{\prime} / z^{\prime}\right) \cap$ $(\Re / z)=Q / z, \operatorname{ht}\left(Q^{\prime} / z^{\prime}\right)=\operatorname{ht}(Q / z)$, by [7, Theorem 3.8], so ht $(Q / z)=1$.

Now, since $R / z_{0}$ satisfies the altitude formula,

$$
\begin{aligned}
\operatorname{ht}(Q / z)+ & \operatorname{trd}\left(((\Re / z) /(Q / z)) /\left(\left(R / z_{0}\right) /\left(P / z_{0}\right)\right)\right) \\
& =\operatorname{ht}\left(P / z_{0}\right)+\operatorname{trd}\left((\Re / z) /\left(R / z_{0}\right)\right) .
\end{aligned}
$$

Now

$$
\operatorname{ht}(Q / z)=1 \text { and } \operatorname{trd}\left((\Re / z) /\left(R / z_{0}\right)\right)=1 \text {, }
$$

since $\mathcal{R} / z \cong \mathcal{R}\left(R / z_{0},\left(I+z_{0}\right) / z_{0}\right)$, so

$$
\operatorname{ht}\left(P / z_{0}\right)=\operatorname{trd}((\Re / Q) /(R / P)) .
$$

Since $R$ is locally quasi-unmixed, $\operatorname{ht}(P)=\operatorname{ht}\left(P / z_{0}\right)$. Let $S=R-P$, so $\Re_{S}=\Re\left(R_{P}, I R_{P}\right)$. Then

$$
\begin{aligned}
\operatorname{alt}\left(\Re_{P}\right)-1 & =\operatorname{alt}\left(R_{P}\right)=\operatorname{ht}(P)=\operatorname{trd}((\Re / Q) /(R / P)) \\
& =\operatorname{trd}\left(\left(\Re_{S} / Q \Re_{S}\right) /\left(R_{P} / P R_{P}\right)\right) .
\end{aligned}
$$

However, $R_{P} / P R_{P} \cong(R / P)_{P / P}$ and

$$
\Re_{S} / Q \Re_{S}=\Re_{(R-P)} / Q \Re_{(R-P)} \cong(\Re / Q)_{(R / P)-(P / P)} ;
$$


so

$$
\operatorname{ht}(P)=\operatorname{trd}\left(\left(\Re_{S} / Q \Re_{S}\right) /\left(R_{P} / P R_{P}\right)\right)=\operatorname{alt}\left(\Re_{S} / Q \Re_{S}\right),
$$

by $[4,(14.6)]$,

$$
\begin{aligned}
& =\operatorname{depth} Q \Re_{S} \\
& \leq \operatorname{depth}(P, u) \Re_{S} \\
& =l\left(I R_{P}\right) \leq \text { alt } R_{P}=\operatorname{ht}(P) .
\end{aligned}
$$

Therefore ht $(P)=l\left(I R_{P}\right)$.

The next corollary extends (2.8) to the case of an arbitrary e.p.f.

(2.9) CoROllary. Let $R$ be a Noetherian ring. Then $R$ is locally quasi-unmixed if and only if for every strong e.p.f. $f=\left\{I_{n}\right\}$ in $R$ and for every prime ideal $P \in \hat{A}^{*}(f), \operatorname{ht}(P)=l\left(f R_{P}\right)$.

Proof. Let $R$ be locally quasi-unmixed and let $f=\left\{I_{n}\right\}$ be a strong e.p.f. in $P$. Assume $P \in \hat{A}^{*}(f)$. By (2.2.4) $\hat{A}^{*}(f)=\hat{A}^{*}\left(I_{n m^{\prime}}\right)$ for every integer $n>0$. From (2.8), $\operatorname{ht}(P)=l\left(I_{n m^{\prime}}, R_{P}\right)$. However, (2.6) shows that $l\left(I_{n m^{\prime}}, R_{P}\right)=l\left(f R_{P}\right)$. Thus ht $(P)=l\left(f R_{P}\right)$.

For the converse, let $I$ be an ideal in $R$ and let $f=\left\{I^{n}\right\}$. Assume $P \in \hat{A}^{*}(f)$, so ht $(P)=l\left(f R_{P}\right)$. By $(2.6), l\left(f R_{P}\right)=l\left(I R_{P}\right)$. Thus ht $(P)=$ $l\left(I R_{P}\right)$, so, by (2.8), $R$ is locally quasi-unmixed.

Our next result, (2.10), together with (2.12) extends a theorem of Ratliff, (cf. [12, Theorem 1]) from the case of an integral domain satisfying the altitude formula, to that of a locally quasi-unmixed Noetherian ring. In (2.11) and (2.13), this is further extended by consideration of arbitrary e.p.f.'s

(2.10) Proposition. The following statements are equivalent for a Noetherian ring $R$ :

(2.10.1) $R$ is locally quasi-unmixed.

(2.10.2) If $I \subseteq P$ are ideals in $R$ such that $P \in \hat{A}^{*}(I)$, then $\operatorname{ht}(P)=$ $l\left(I R_{P}\right)$.

(2.10.3) If $I$ is an ideal in $R$ and $P$ is a prime divisor of $\left(I^{n}\right)_{a}$ for some $n \geq 1$, then $\mathrm{ht}(P)=l\left(I R_{P}\right)$.

(2.10.4) If $I$ is an ideal in $R$ such that $\mathrm{ht}(I)=V(I)$, then $\left(I^{n}\right)_{a}$ is height unmixed for every integer $n \geq 1$. 
Proof. (2.10.1) is equivalent to (2.10.2) by (2.8). (2.10.2) and (2.10.3) are equivalent, by $[9,(2.5)]$. Finally, $(2.10 .1)$ and $(2.10 .4)$ are equivalent by $[8,(2.29)]$,

(2.11) COROLlaRY. The following statements are equivalent for a Noetherian ring $R$;

(2.11.1) $R$ is locally quasi-unmixed.

(2.11.2) For every strong e.p.f. $f=\left\{I_{n}\right\}$ in $R$, if $P$ is a prime divisor of $\left(I_{n}\right)_{\alpha}$ for some $n \geq 1$, then $\mathrm{ht}(P)=l\left(f R_{P}\right)$.

(2.11.3) For every strong e.p.f. $f=\left\{I_{n}\right\}$ in $R$ and for each fixed $n \geq 1$, if $\mathrm{ht}\left(I_{n m^{\prime}}\right)=V\left(I_{n m^{\prime}}\right)$, then $\left(I_{n m^{\prime}}\right)_{\alpha}$ is height unmixed.

Proof. By [6, (3.6.1)] if $f$ is an e.p.f. in $R$ and if $P \in \hat{A}(k)$ for some $k>0$, then $P \in \hat{A}(n)$ for all large $n$. Thus if $P \in \hat{A}(n)$ for some $n>1$, then $P \in \hat{A}^{*}(f)$. Therefore, by (2.9), (2.11.1) and (2.11.2) are equivalent.

Next we show (2.11.2) implies (2.11.3). So assume (2.11.2) holds, let $f$ be a strong e.p.f. in $R$, fix $n \geq 1$, let $h t\left(I_{n m^{\prime}}\right)=v\left(I_{n m^{\prime}}\right)$, and let $P \in$ $\operatorname{Ass}\left(\left(I_{n m^{\prime}}\right)_{\alpha}\right)$. Then, by (2.11.2), $\operatorname{ht}(P)=l\left(f R_{P}\right)$, and (2.6) implies that $l\left(f R_{P}\right)=l\left(I_{n m^{\prime}} R_{P}\right)$. Now we have

$$
\operatorname{ht}(P)=l\left(I_{n m^{\prime}} R_{P}\right) \leq v\left(I_{n m^{\prime}}\right)=\operatorname{ht}\left(I_{n m^{\prime}}\right) \leq \mathrm{ht}(P),
$$

since $l\left(I_{n m^{\prime}} R_{P}\right) \leq v\left(I_{n m^{\prime}}\right)$, by (2.4). Therefore $\operatorname{ht}(P)=\operatorname{ht}\left(I_{n m^{\prime}}\right)$ and so $\left(I_{n m^{\prime}}\right)_{\alpha}$ is height unmixed. This shows (2.11.2) implies (2.11.1).

Finally, let $I$ be an ideal in $R$ such that $\operatorname{ht}(I)=v(I)$ and let $f=\left\{I^{n}\right\}$. Then $m^{\prime}=1$, so (2.11.3) says that $\left(I^{n}\right)_{a}=\left(I^{n}\right)_{\alpha}$ is height unmixed. Thus, by $(2.10 .4) \Rightarrow(2.10 .1), R$ is locally quasi-unmixed, and so $(2.11 .3) \Rightarrow$ (2.11.1).

It will now be shown that the equivalent conditions in (2.10) imply the converse of (2.10.2). As already noted, this generalizes [13, Theorem 1].

(2.12) Proposition. Let $R$ be a locally quasi-unmixed Noetherian ring and let $I \subseteq P$ be ideals in $R$ with $P$ prime. If $\mathrm{ht}(P)=l\left(I R_{P}\right)$, then $P \in \hat{A}^{*}(I)$.

Proof. The proof is the same as the proof for the corresponding result in [13, Theorem 1], except for the last sentence: Then $Q$ is a prime divisor of $(u \Re)_{a}$, so the proof of (2.8.1) shows $Q$ is a prime divisor of $\left(I_{n}\right)_{\alpha}=\left(I^{n}\right)_{a}$ for all large $n$, and so $P \in \hat{A}^{*}(I)$, by (2.2.3). 
We now extend (2.12) to the case of an arbitrary e.p.f.

(2.13) Corollary. Let $R$ be a locally quasi-unmixed Noetherian ring, let $f=\left\{I_{n}\right\}$ be a strong e.p.f. in $R$, and let $P$ be a prime ideal in $R$ such that $f \subseteq P$. If $\operatorname{ht}(P)=l\left(f R_{P}\right)$, then $P \in \hat{A}^{*}(f)$.

Proof. Let ht $(P)=l\left(f R_{P}\right)$. Then, by (2.6), ht $(P)=l\left(I_{n m^{\prime}} R P\right)$ for all integers $n \geq 1$. Therefore, by (2.12), $P \in \hat{A}^{*}\left(I_{n m^{\prime}}\right)$ for all $n \geq 1$, so $P \in$ $\hat{A}^{*}(f)$ by (2.2.4).

(2.14) gives three more conditions which are equivalent to a Notherian ring $R$ being locally quasi-unmixed. (2.14) generalizes [12, Theorem 2] from the case of a Noetherian domain to that of a locally quasi-unmixed Noetherian ring.

(2.14) Proposition. The following statements are equivalent for a Noetherian ring $R$.

(2.14.1) $R$ is locally quasi-unmixed.

(2.14.2) If $I \subseteq M$ are ideals in $R$ such that $M$ is maximal, $\mathrm{ht}(I)=V(I)$ and $\operatorname{ht}(M / I)=1$, then, for every $n \geq 1, M$ is not a prime divisor of $\left(I^{n}\right)_{a}$.

(2.14.3) If $I \subseteq M$ are ideals in $R$ with $M$ maximal such that ht $(M / I)$ $=1$ and if $I$ is integrally dependent on an ideal $B$ of the principal class, then, for every integer $n \geq 1, M$ is not a prime divisor of $\left(I^{n}\right)_{a}$.

(2.14.4) If $I$ is an ideal in $R$ which can be generated by $h$ elements, then, for every integer $n \geq 1,\left(I^{n}\right)_{a}$ has no prime divisors of height strictly greater than $h$.

Proof. (2.14.1) and (2.14.2) are equivalent by [8, (2.29)]. It is clear that (2.14.3) implies (2.14.2). We now show that (2.14.2) implies (2.14.3). For this, let $I \subseteq M$ be ideals in $R$ such that $\mathrm{ht}(M / I)=1$ and assume $B$ is an ideal of the principal class such that $I$ is integrally dependent on $B$. It suffices to show that $M$ is not a prime divisor of $\left(B^{n}\right)_{a}$ for $n \geq 1$, since $B \subseteq I \subseteq B_{a}$ implies $\left(B^{n}\right)_{a}=\left(I^{n}\right)_{a}$ for all $n \geq 1$. To insure that $B \subseteq I \subseteq M$ satisfies the hypothesis of (2.14.2), we need only show that $h \mathrm{t}(M / B)=1$. But this holds, since $B \subseteq I \subseteq(B)_{a} \subseteq \operatorname{rad}(B)$. Thus (2.14.2) implies (2.14.3).

Next it will be shown that (2.14.1) and (2.14.4) are equivalent. To see that (2.14.4) implies (2.14.1), it suffices, by [8, (2.29)], to show that for every ideal $I$ of the principal class, $\left(I^{n}\right)_{a}$ is height unmixed for $n \geq 1$. Let $I$ be an ideal in $R$ such that ht $(I)=V(I)$ and let $P$ be a prime divisor of 
$\left(I^{n}\right)_{a}$ for some $n \geq 1$. Then, by (2.14.4), $h=V(I)=\operatorname{ht}(I)=\operatorname{ht}\left(I^{n}\right)_{a} \leq$ $\mathrm{ht}(P) \leq h$, so $\mathrm{ht}(P)=V(I)$ and $\left(I^{n}\right)_{a}$ is height unmixed. Thus $R$ is locally quasi-unmixed, so (2.14.4) implies (2.14.1).

Finally, assume $R$ is locally quasi-unmixed and let $I$ be an ideal in $R$ such that $I$ is generated by $h$ elements. Let $P$ be a prime divisor of $\left(I^{n}\right)_{a}$ for some integer $n \geq 1$. Then by $(2.10 .1) \Leftrightarrow(2.10 .3), \operatorname{ht}(P)=l\left(I R_{P}\right) \leq$ $V\left(I R_{P}\right) \leq V(I) \leq h$. Thus (2.14.1) implies (2.14.4).

(2.15) generalizes (2.14) to the case of an arbitrary e.p.f..

(2.15) COROLlaRY. The following statements are equivalent for a Noetherian ring $R$ :

(2.15.1) $R$ is locally quasi-unmixed.

(2.15.2) If $f=\left\{I_{n}\right\}$ is an e.p.f. in $R$ and $M$ is a maximal ideal in $R$ such that $f \subseteq M, \operatorname{ht}\left(I_{m^{\prime}}\right)=v\left(I_{m^{\prime}}\right)$, and if $\operatorname{ht}\left(M / I_{m^{\prime}}\right)=1$, then, for all large $n, M$ is not a prime divisor of $\left(I_{n}\right)_{\alpha}$.

(2.15.3) If $f=\left\{I_{n}\right\}$ is a strong e.p.f. in $R$ and $M$ is a maximal ideal in $R$ such that $f \subseteq M, \operatorname{ht}\left(M / I_{m^{\prime}}\right)=1$, and $I_{m^{\prime}}$ is weakly integrally dependent on an ideal $B$ of the principal class, then, for all large $n, M$ is not a prime divisor of $\left(I_{n}\right)_{\alpha}$.

(2.15.4) If $f=\left\{I_{n}\right\}$ is a strong e.p.f. in $R$ such that $I_{m^{\prime}}$ is generated by $h$ elements, then, for all large $n,\left(I_{n}\right)_{\alpha}$ has no prime divisors of height strictly greater than $h$.

Proof. Assume $R$ is locally quasi-unmixed, and let $f$ and $M$ satisfy the hypothesis of (2.15.2). Then, by $(2.14 .1) \Rightarrow(2.14 .2), M$ is not a prime divisor of $\left(I_{k m^{\prime}}\right)_{\alpha}=\left(\left(I_{m^{\prime}}\right)^{k}\right)_{a}$. But, for large $k$ and $n$, Ass $\left(\left(I_{k m^{\prime}}\right)_{a}\right)=$ $\operatorname{Ass}\left(\left(I_{n}\right)_{\alpha}\right)=\hat{A}^{*}$, by (2.2.4). Thus (2.15.1) implies (2.15.2).

(2.15.2) implies (2.15.1), by (2.14.2) implies (2.14.1). It is clear that (2.15.3) implies (2.15.2). For the converse, let $M, f$, and $B$ be as in (2.15.3). Then, by (2.14.3), $M$ is not a prime divisor $\left(\left(I_{m^{\prime}}\right)^{k}\right)_{a}=\left(I_{k m^{\prime}}\right)_{\alpha}$, for $k \geq 1$. Since, by (2.2.3) and (2.2.4), $\operatorname{Ass}\left(\left(I_{k m^{\prime}}\right)_{\alpha}\right)=\operatorname{Ass}\left(\left(I_{n}\right)_{\alpha}\right)=\hat{A}^{*}$, for large $n$ and $k, M$ is not a prime divisor of $\left(I_{n}\right)_{\alpha}$ for all large $n$. Therefore (2.15.2) implies (2.15.3).

(2.15.4) implies (2.15.1), by, (2.11.3) $\Rightarrow(2.11 .1)$. To show (4.13.1) implies (2.15.4), assume that $R$ is locally quasi-unmixed and let $f=\left\{I_{n}\right\}$ be an e.p.f. in $R$ such that $I_{m^{\prime}}$ is generated by $h$ elements. Then $\left(\left(I_{m^{\prime}}\right)^{k}\right)_{a}=$ $\left(I_{m^{\prime} k}\right)_{\alpha}$ has no prime divisor of height $>h$, by $(2.14 .1) \Rightarrow(2.14 .4)$. Therefore, since $\left(I_{n}\right)_{\alpha}$ and $\left(I_{m^{\prime} k}\right)_{\alpha}$ have the same prime divisors for large $n$ and $k$, by (3.9), (2.15.1) $\Rightarrow(2.15 .4)$. 
(2.16.1) is a corollary to (2.8) and (2.12). (2.16.2) gives a condition for the analytic spread of an ideal to be equal to its height. (2.16) is extended to the case of an e.p.f. in (2.17).

(2.16) Proposition. (C.f. [12, Corollary 4].) Let $R$ be a locally quasi-unmixed Noetherian ring and let $I \subseteq P$ be ideals in $R$ such that $P$ is a prime ideal. Then the following statements are true:

(2.16.1) $P \in \hat{A}^{*}(I)$ if and only if $\mathrm{ht}(P)=l\left(I R_{P}\right)$

(2.16.2) Assume $R$ is local with maximal ideal $P$. If $\mathrm{ht}(P / I)=1$, then $\operatorname{ht}(I)=l(I)=\operatorname{alt}(R)-1$ if and only if $P \notin \hat{A}^{*}(I)$.

Proof. For (2.16.1), if $P \in \hat{A}^{*}(I)$, then $\mathrm{ht}(P)=l\left(I R_{P}\right)$, by (2.8), and if $\operatorname{ht}(P)=l\left(I R_{P}\right)$ then (2.12) implies $P \in \hat{A}^{*}(I)$.

To prove (2.16.2), assume ht $(P / I)=1$. Let ht $(I)=l(I)=\operatorname{alt}(R)-$ 1 and suppose $P \in \hat{A}^{*}(I)$. Then, by (2.16.1), ht $(P)=l(I)$. But $\operatorname{ht}(P)>$ $\operatorname{ht}(I)=l(I)$, so this contradiction shows $P \notin \hat{A}^{*}(I)$. For the converse, assume $p \notin \hat{A}^{*}(I)$. Suppose $\operatorname{depth}(I)=1, \operatorname{ht}(I)=\operatorname{alt}(R)-1$, by $[4$, (34.5)]. Also, $\operatorname{ht}(P)>l(I)$, by hypothesis and (2.12), so $P$ is the maximal ideal in $R$. Thus $l(I) \leq \mathrm{ht}(P)-1=\mathrm{ht}(I)$. But, there exists a prime divisor $Q$ of $I$ such that $\operatorname{ht}(Q)=\operatorname{ht}(I)$. Then $Q \in \hat{A}^{*}(I)$, and so ht $(I)=$ $\operatorname{ht}(Q)=l\left(I R_{Q}\right) \leq l(I)$ by $([12$, comments preceding Corollary 9] $) \leq$ $\operatorname{ht}(I)$. Therefore $\operatorname{ht}(I)=l(I)$. Therefore $\operatorname{ht}(I)=l(I)=\operatorname{alt}(R)-1$, so (2.16.2) holds.

(2.17) COROllaRY. Let $R$ be a locally quasi-unmixed Noetherian ring, let $f=\left\{I_{n}\right\}$ be a strong e.p.f. in $R$ and let $P$ be a prime ideal in $R$ such that $I_{1} \subseteq P$. Then the following statements are true:

(2.17.1) $P \in \hat{A}^{*}(f)$ if and only if $\mathrm{ht}(P)=l\left(f R_{P}\right)$.

(2.17.2) Assume $R$ is local with maximal ideal $P$. If $\mathrm{ht}\left(P / I_{m^{\prime}}\right)=1$, then $\operatorname{ht}(f)=l(f)=\operatorname{alt}(R)-1$ if and only if $P \notin \hat{A}^{*}(f)$.

Proof. (2.17.1) is immediate from (2.9) and (2.13).

For (2.17.2), assume ht $\left(P / I_{m^{\prime}}\right)=1$ and let $\operatorname{ht}(f)=l(f)=\operatorname{alt}(R)-$ 1. Then, since $\operatorname{ht}(f)=\operatorname{ht}\left(I_{m^{\prime}}\right)$ and $l(f)=l\left(I_{m^{\prime}}\right)$ (by (2.6)), $P \notin \hat{A}^{*}\left(I_{m^{\prime}}\right)$, by (2.8), and so $P \notin \hat{A}^{*}(f)$. For the converse, assume $P \notin \hat{A}^{*}(f)$, so $P \notin \hat{A}^{*}\left(I_{m^{\prime}}\right)$, by (2.2.3). By (2.16.2), $\operatorname{ht}\left(I_{m^{\prime}}\right)=l\left(I_{m^{\prime}}\right)=\operatorname{alt}(R)-1$, and (2.17.2) readily follows from this.

We conclude this section with three interesting applications of (2.8) and (2.14). 
(2.18) COROllary. (Cf. [12, Corollary 6].) Let $R$ be a Noetherian ring and let $M$ be a maximal ideal in $R$. Then $R_{M}$ is quasi-unmixed if and only if there does not exist a system of parameters $b_{1}, \ldots, b_{d}$ in $R_{M}$ such that $M R_{M}$ is a prime divisor of $\left(\left(b_{1}, \ldots, b_{d-1}\right)^{n}\right)_{a}$ for some integer $n>0$.

Proof. Assume $R_{M}$ is quasi-unmixed (hence locally quasi-unmixed), and let $b_{1}, \ldots, b_{d}$ be a system of parameters in $R_{M}$. Then $\operatorname{ht}\left(b_{1}, \ldots, b_{d-1}\right)$ $=d-1$, by $[3,(12 . \mathrm{K})]$ and $[4,(34.5)]$. Therefore, for each $n \geq 1, M R_{M}$ is not a prime divisor of $\left(\left(b_{1}, \ldots, b_{d-1}\right)^{n}\right) a$ by $(2.14 .1) \Rightarrow(2.14 .2)$.

For the reverse implication assume there does not exist a system of parameters $b_{1}, \ldots, b_{d}$ in $R_{M}$ such that $M R_{M}$ is a prime divisor of $\left(\left(b_{1}, \ldots, b_{d-1}\right)^{n}\right)_{a}$ for some $n>0$. Then it readily follows from $(2.14 .2) \Rightarrow$ (2.14.1) that $R_{M}$ is quasi-unmixed.

(2.19) is essentially a restatement of (2.18).

(2.19) COROLlary. Let $R$ be a Noetherian ring and let $M$ be a maximal ideal in $R$. Then $R_{M}$ is quasi-unmixed if and only if there does not exist a system of parameters $b_{1}, \ldots, b_{d}$ in $R_{M}$ such that $M R_{M} \in \hat{A}^{*}\left(b_{1}, \ldots, b_{d-1}\right)$.

Proof. This follows immediately form (2.18) and [9, (2.5)].

This chapter will be closed with the following corollary.

(2.20) Corollary. Let $(R, M)$ be a quasi-unmixed local ring, let $\operatorname{alt}(R)=d \geq 1$, and let $I$ be an ideal in $R$ such that $\mathrm{ht}(I)<d$. Then the following statements are true:

(2.20.1) $M \notin \hat{A}^{*}(I)$ if and only if $l(I)<d$.

(2.20.2) If there exists a prime ideal $P \in \hat{A}^{*}(I)$ such that $\mathrm{ht}(I)=\operatorname{ht}(P)$ and $l(I)=l(I R p)$, then $\mathrm{ht}(I)=l(I)$.

(2.20.3) If $\mathrm{ht}(I)=l(I)$, then, for each $n \geq 1$, all prime divisors of $\left(I^{n}\right)_{a}$ have the same height.

Proof. We first prove (2.20.1). From (2.16.1), $M \notin \hat{A}^{*}(I)$ if and only if $\operatorname{ht}(M) \neq l\left(I R_{M}\right)$. However, it is clear that $\mathrm{ht}(M) \neq l\left(I R_{M}\right)$ if and only if $l(I)<d$, so $(2.20 .1)$ holds.

For (2.20.2), let $P \in \hat{A}^{*}(I)$ such that $\operatorname{ht}(I)=\mathrm{ht}(P)$ and $l(I)=$ $l\left(I R_{P}\right)$. Then, by $(4.6), \operatorname{ht}(P)=l\left(I R_{p}\right)$, so $\mathrm{ht}(I)=\mathrm{ht}(P)=l\left(I R_{P}\right)=$ $l(I)$, proving (2.10.2).

To see (2.20.3), assume $h \mathrm{t}(I)=l(I)$. Let $n \geq 1$ and let $P \in$ $\operatorname{Ass}\left(\left(I^{n}\right)_{a}\right)$. Then ht $(I) \leq \mathrm{ht}(P)=l\left(I R_{P}\right) \leq l(I)=\mathrm{ht}(I)$. Thus, for every integer $n \geq 1,\left(I^{n}\right)_{a}$ is height unmixed. 


\section{REFERENCES}

[1] W. Bishop, A theory of multiplicity for multiplicative filtrations, Ph.D. Dissertation, Western Michigan Univ. 1971.

[2] P. Eakin and S. McAdam, The asymptotic Ass, J. Algebra, 61 (1979), 71-81.

[3] Matsumura, Commutative Algebra, W. A. Benjamin, Inc., New York, N. Y., 1970.

[4] M. Nagata, Local Rings, Interscience Publishers, New York, N. Y., 1962.

[5] J. S. Okon, Asymptotic prime divisors and filtrations, Ph.D. Dissertation, University of California, Riverside, 1980.

[6] _ Prime divisors and filtrations, 27 page preprint.

[7] L. J. Ratliff, Jr., On quasi-unmixed local domains, the altitude formula, and the chain condition for prime ideals (I), Amer. J. Math., 41 (1969), 508-528.

[8] Locally quasi-unmixed Noetherian rings and ideals of the principal class, Pacific J. Math., 52 (1974), 185-205.

[9] _ On prime divisors of $I^{n}$, $n$ large, Michigan Math. J., 23 (1976), 337-352.

[10] _ Notes on essentially powers filtrations, Michigan Math. J., 26 (1979), 313-324.

[11] Note on asymptotic prime divisors, analytic spreads, and the altitude formula, Proc. of the Amer. Math. Soc., 82 (1981), 1-6.

[12] _ Integrally closed ideals and asymptotic prime divisors, Pacific J. Math., 91 (1980), 445-456.

[13] D. Rees, Form rings and ideals, Mathematica, 4 (1957), 51-60.

Received November 11, 1982

California State College

SAN BERNARDino, CA 92407 


\section{PACIFIC JOURNAL OF MATHEMATICS \\ EDITORS}

Donald BaBBITT (Managing Editor)

University of California

Los Angeles, CA 90024

Hugo RossI

University of Utah

Salt Lake City, UT 84112

C. C. Moore and Arthur Ogus

University of California

Berkeley, CA 94720
J. DugundiI

Department of Mathematics

University of Southern California

Los Angeles, CA 90089-1113

R. FINN and H. SAMELSON

Stanford University

Stanford, CA 94305

\section{ASSOCIATE EDITORS}
R. ARENS
E. F. BECKENBACH
B. H. NeUMANN
F. WOLF
K. YoshidA (1906-1982)

\section{SUPPORTING INSTITUTIONS}

\author{
UNIVERSITY OF ARIZONA \\ UNIVERSITY OF BRITISH COLUMBIA \\ CALIFORNIA INSTITUTE OF TECHNOLOGY \\ UNIVERSITY OF CALIFORNIA \\ MONTANA STATE UNIVERSITY \\ UNIVERSITY OF NEVADA, RENO \\ NEW MEXICO STATE UNIVERSITY \\ OREGON STATE UNIVERSITY
}

\author{
UNIVERSITY OF OREGON \\ UNIVERSITY OF SOUTHERN CALIFORNIA \\ STANFORD UNIVERSITY \\ UNIVERSITY OF HAWAII \\ UNIVERSITY OF TOKYO \\ UNIVERSITY OF UTAH \\ WASHINGTON STATE UNIVERSITY \\ UNIVERSITY OF WASHINGTON
}

The Supporting Institutions listed above contribute to the cost of publication of this Journal, but they are not owners or publishers and have no responsibility for its content or policies.

Mathematical papers intended for publication in the Pacific Journal of Mathematics should be in typed form or offset-reproduced (not dittoed), double spaced with large margins. Please do not use built up fractions in the text of the manuscript. However, you may use them in the displayed equations. Underline Greek letters in red, German in green, and script in blue. The first paragraph must be capable of being used separately as a synopsis of the entire paper. In particular it should contain no bibliographic references. Please propose a heading for the odd numbered pages of less than 35 characters. Manuscripts, in triplicate, may be sent to any one of the editors. Please classify according to the scheme of Math. Reviews, Index to Vol. 39. Supply name and address of author to whom proofs should be sent. All other communications should be addressed to the managing editor, or Elaine Barth, University of California, Los Angeles, California 90024.

There are page-charges associated with articles appearing in the Pacific Journal of Mathematics. These charges are expected to be paid by the author's University, Government Agency or Company. If the author or authors do not have access to such Institutional support these charges are waived. Single authors will receive 50 free reprints; joint authors will receive a total of 100 free reprints. Additional copies may be obtained at cost in multiples of 50 .

The Pacific Journal of Mathematics is issued monthly as of January 1966. Regular subscription rate: $\$ 132.00$ a year (6 Vol., 12 issues). Special rate: $\$ 66.00$ a year to individual members of supporting institutions.

Subscriptions, orders for numbers issued in the last three calendar years, and changes of address should be sent to Pacific Journal of Mathematics, P.O. Box 969, Carmel Valley, CA 93924, U.S.A. Old back numbers obtainable from Kraus Periodicals Co., Route 100, Millwood, NY 10546.

The Pacific Journal of Mathematics ISSN 0030-8730 is published monthly by the Pacific Journal of Mathematics at P.O. Box 969, Carmel Valley, CA 93924. Application to mail at Second-class postage rates is pending at Carmel Valley, California, and additional mailing offices. Postmaster: Send address changes to Pacific Journal of Mathematics, P. O. Box 969, Carmel Valley, CA 93924.

PUBLISHED BY PACIFIC JOURNAL OF MATHEMATICS, A NON-PROFIT CORPORATION

Copyright $\odot 1984$ by Pacific Journal of Mathematics 


\section{Pacific Journal of Mathematics}

\section{Vol. 113, No. $2 \quad$ April, 1984}

Alan Adolphson, On the Dwork trace formula ...................257

Amos Altshuler and Leon Steinberg, Enumeration of the quasisimplicial

3 -spheres and 4-polytopes with eight vertices .................. 269

Kenneth R. Goodearl, Cancellation of low-rank vector bundles .......... 289

Gary Fred Gruenhage, Ernest A. Michael and Yoshio Tanaka, Spaces

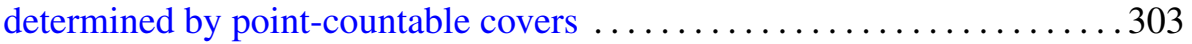

Charles Lemuel Hagopian, Atriodic homogeneous continua .......... 333

David Harbater, Ordinary and supersingular covers in characteristic $p$. . . 349

Domingo Antonio Herrero, Continuity of spectral functions and the lakes

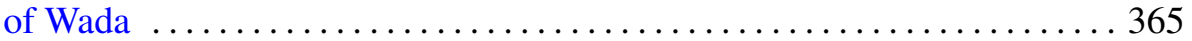

Donald William Kahn, Differentiable approximations to homotopy resolutions and framed cobordism ....................... 373

K. McGovern, On the lifting theory of finite groups of Lie type $\ldots \ldots \ldots . \ldots 383$

C. David (Carl) Minda, The modulus of a doubly connected region and the geodesic curvature-area method ............................. 395

Takuo Miwa, Complexes are spaces with a $\sigma$-almost locally finite base $\ldots . .407$

Ho Kuen Ng, Finitely presented dimension of commutative rings and modules

Roger David Nussbaum, A folk theorem in the spectral theory of

$C_{0}$-semigroups

J. S. Okon, Prime divisors, analytic spread and filtrations

Harold Raymond Parks, Regularity of solutions to elliptic isoperimetric problems

R. Sitaramachandra Rao and M. V. Subba Rao, Transformation formulae for multiple series

Daniel Ruberman, Imbedding punctured lens spaces and connected sums

Uri Srebro, Deficiencies of immersions 